Rapid Reviews COVID-19

\title{
Review 2: "Medical Costs of Keeping the US Economy Open During COVID-19"
}

Mehdi Shiva ${ }^{1}$

${ }^{1}$ Blavatnik School of Government

Published on: Aug 20, 2020

DOI: 10.1162/2e3983f5.8b63288a

License: Creative Commons Attribution 4.0 International License (CC-BY 4.0). 


\section{$\underline{\text { RR:C19 Evidence Scale rating by reviewer: }}$}

- Potentially informative. The main claims made are not strongly justified by the methods and data, but may yield some insight. The results and conclusions of the study may resemble those from the hypothetical ideal study, but there is substantial room for doubt. Decision-makers should consider this evidence only with a thorough understanding of its weaknesses, alongside other evidence and theory. Decisionmakers should not consider this actionable, unless the weaknesses are clearly understood and there is other theory and evidence to further support it.

$* * * * * * * * * * * * * * * * * * * * * * * * * * * * * * * * * * * * * * *$

\section{Review:}

The following is a review of "Medical Costs of Keeping the US Economy Open During COVID-19". The authors use an individual level synthetic social contact network based on publicly available data and national level epidemic simulations to estimate the medical costs of keeping the US economy open under COVID-19 pandemic. Amongst a range of findings, the simulations suggest that the US economy could be brought down by $5 \%$ if no mitigation strategies were taken.

Overall, a valid approach and high-quality analysis were conducted. The study is topical and relevant to the policy as well. The scope of this paper is limited to medical costs, and not the economic cost, of the pandemic and therefore has a limited role in practice. This is somehow acknowledged by the authors. There are also some critics around using value of statistical life (VSL) in COVID-19 cost simulations, e.g. Adler (2020) states in COVID Economics volume 33 “... VSL is only an approximation to individual willingness to pay, which may become quite inaccurate for policies that mitigate large risks (such as the risks posed by COVID-19) - and thus can recommend policies that fail the Kaldor-Hicks test."

However, the main concerns are around the assumptions that are taken either implicitly or explicitly in this study which might affect the robustness of findings negatively. I believe addressing the following points could improve the policy relevance of the paper. The first two comments require minor-adjustments and a re-run of the simulations, where the third one could be simply added to the limitations section.

1. Based on OECD and on average, $65 \%$ of total beds in the US are occupied at any time (this is $80 \%$ for Europe). Even if hospitals manage to free up some of the beds 
(sending patients home or not admiting new patients after discharge), lets say by 30\%, there are still atleast $40 \%$ of beds occupied at any point. So the sensitivity analysis of bed availability between 70\%-120\% might seems unrealistic. I recommend trying a different range, maybe around 30\%-90\%. This is an important consideration given that even the authors state that " $A$ sensitivity analysis of the length of hospital stay, and dedicated bed capacity available to COVID-19 patients, shows that the number of deaths depend significantly on these parameters" (page 11). Given that 'death' is the main driver of the estimated costs in this paper, as it is priced at a substantially higher price than any other health state, even small changes to these rates should change the final figures substantially.

2. Stay home is a mitigation strategy but not an applicable policy. Government containment policies include workplace closure, school closure, etc. To my understanding, no country has ever taken a full lockdown or stay home policy which includes essential workers as well. It might be unrealistic to achieve a $70 \%$ or above of the total population - compliance rate given that a considerable share of the workforce in developed countries are essential; e.g. workers deemed essential by Department of Homeland Security vary between 18.6\% to 45\% across the 192 metro areas - based on Brookings (Tomer and Kane, 2020). Also, Economic Policy Institute (McNicholas and Poydock, 2020) estimates 55 million essential workers in the US (around $43 \%$ of the total workforce). It would be good to consider such limitations in modelling.

3. exclusion of Indirect medical costs is definitely a limitation. The consequences of over-loading hospital beds with COVID-patients is substantial on other patients and could potentially even increase their mortality rates. In a developed country setting, David Miles (2020) states in a VOX EU column "Health costs from telling people to stay at home are likely to be large and long-lasting. Referrals for cancer investigations were 70\% down in April 2020; there were hardly any follow-up routine appointments for long-term conditions in UK primary care between mid-March 2020 and the beginning of June; outpatients seen were $64 \%$ down and elective admissions to hospitals down 75\%; attended appointments in general practice were down 35\%. The impact of the stress of the lockdown on anyone with a pre-existing mental health condition, let alone the population as a whole, is yet to be determined". another example of the burden of the indirect effects in developing countries is Roberton et al. (2020) in Lancet Glob Health. 